\title{
TELPACK: An Advanced Teletraffic Analysis Package
}

Nail Akar, Sprint

Nihat Cem Oguz and Khosrow Sohraby, University of Missouri-Kansas City

Performance evaluation of computer and communication net-
works gives rise to teletraffic problems of potentially large
dimensionality. We briefly summarize a unifying system theoretic approach to efficient
solution of a diversity of such problems, and introduce TELPACK, a publicly available soft-
ware that implements this approach.

from the former. The approach outlined here is based on invariant subspaces, and uses two very mature fields of applied mathematics, namely linear

D erformance evaluation problems arising in the study of telecommunication networks basically consist of two components: traffic modeling and queuing analysis. Traffic is the driving force behind all telecommunications activity, and its models are of crucial importance for evaluating network performance [1]. Probability models for traffic streams are needed to the extent that they allow prediction of system performance measures to a reasonable degree of accuracy. The fundamental systems in telecommunication networks, of which traffic is the major constituent, are queuing systems, and their numerical analysis provides various system performance measures if a suitable traffic model is available.

Consider a high-speed packet-switched network, such as asynchronous transfer mode (ATM) or integrated services Internet, in which several multimedia sources are statistically multiplexed over the network links. Design and engineering of such networks to provide quality of service guarantees to individual sources in terms of connection blocking probabilities, loss rates, and delays requires development of traffic models at call and cell levels. These models are very useful to tackle the fundamental problems of networking, namely, link dimensioning, bandwidth enforcement, call admission control, and routing.

However, traffic modeling based on traditional Poisson arrival and exponential call holding time assumptions, which have performed successfully in the design and analysis of circuit-switched telephone networks, are no longer valid for high-speed packet-switched networks carrying LAN-to-LAN data, compressed video, and so on. The reason is that such traffic exhibits nontrivial higher-order statistics which are difficult to measure and characterize. In cases when characterization is possible, the probability models to represent the actual traffic may have large dimensionality. For example, the number of states in a Markovian model to characterize an aggregation of several multimedia sources is typically very large. Such potentially large model dimensionality imposes the need for computationally efficient queuing analysis techniques and tools to design, engineer, and dimension networks.

Due to the above, we attempt to provide a unified and systematic way of numerically solving a large class of queuing problems which are frequently encountered in practice. Most of the existing methods are problem-specific, and it is in general hard, if not impossible, to apply a method proposed for one queuing system to another even when the latter is derived

This work was partially supported by DARPA/ITO under grant AO-F316 and by NSF under grant NCR-950814. systems theory and numerical linear algebra, to analyze:

- Continuous-/discrete-time

- Single-/multiple-server

- Finite/infinite queues

in a unified framework while maintaining computational efficiency, robustness, and stability. In most cases where very complex queuing models are encountered, simulation techniques are successfully employed and sometimes preferred to analysis due to the intractability of such models. However, there is a certain need for analytical results when possible to get deeper insight, reduce runtimes, handle very rare events, and optimize system performance. Motivated by this need and the unifying nature of the invariant subspace approach, we have developed a teletraffic analysis package (TELPACK). The current version of TELPACK, which is publicly available [2], implements the invariant subspace approach for analyzing a set of common and important queuing systems. In its future releases, we intend to expand its capabilities and improve ease of use for researchers, practitioners, and students.

In the following, we briefly describe the queuing systems that are of central interest in teletraffic analysis, and introduce the invariant subspace approach along with an overview of traditional approaches. We also highlight some features of the current and future versions of TELPACK.

\section{QUEUING SYSTEMS}

Let us begin our discussion on queuing systems with discretetime queues described by the evolution equation

$$
Q_{n+1}=\min \left[K,\left(Q_{n}+A_{n}-B_{n}\right)^{+}+C_{n}\right]
$$

where (Fig. 1)

$(\cdot)^{+} \triangleq \max (0, \cdot)$

$Q_{n}=$ queue occupancy at time $n$

$K=$ queue storage capacity

$A_{n}, B_{n}, C_{n}=$ all Markovian processes taking nonnegative values

The evolution equation, Eq. 1, is generic in the sense that it covers a wide variety of discrete-time queuing models which have attracted the attention of many researchers in performance evaluation of telecommunication networks.

- When $K \rightarrow \infty$, we have an infinite queue. While infinite queues appear to lack physical justification due to limited storage capacities in real systems, they usually serve as an effective approximation to the case of finite but large buffer sizes. Infinite models have especially been used in the analysis of asymptotic queue length behavior which is closely linked with effective bandwidth computations for call admission control of guaranteed services such as 
variable bit rate (VBR) in ATM networks [3, 4]. The case of finite $K$ is especially interesting in the analysis of unrare losses for best-effort traffic in ATM networks via the use of the ATM Forum service category unspecified bit rate (UBR).

- When $B_{n} \leq 1$, one has an M/G/1 type Markov chain [5], a model which has been used extensively in performance evaluation of ATM multiplexers. When $B_{n}>1$ is possible, one can also study multiple servers, a case which arises particularly in computer systems as well as circuitswitched networks.

- When one of $A_{n}$ or $C_{n}$ is less than or equal to one and the other is zero, the resulting queue can be solved in the framework of G/M/1 type Markov chains [6]. Such chains have proven to be very useful in the performance assessment of computer and communication systems.

- One has a quasi-birth-and-death (QBD) chain when the resulting Markov chain is of both $\mathrm{M} / \mathrm{G} / 1$ and $\mathrm{G} / \mathrm{M} / 1$ types. Due to the relative simplicity of their analysis, QBD chains deserve a particular attention.

- In the most general case, if the three Markovian arrival and service processes in the evolution equation, Eq. 1, are general, the resulting chain is referred to as a blockstructured Markov chain with repeating rows. With this generic structure, one can analyze continuous-time systems including GI/G/1 and semi-Markovian queues as well as discrete-time queues.

When it comes to the analyses of the queuing systems just discussed, we are more interested in the stationary probabilities

$$
q_{k} \triangleq \operatorname{Prob}\left\{Q_{n}=k\right\}, k \geq 0, \text { as } n \rightarrow \infty
$$

than the transient behavior of the queue, although the latter may be helpful in addressing interesting performance problems.

\section{Methods of QueUING AnAlysis}

There are basically three approaches that have been proposed to obtain the stationary solution of queues discussed above. In the transform approach, one first obtains an expression for the generating function of the state probabilities in terms of an unknown vector of coefficients. The second step is to determine the zeros of the determinant of a certain polynomial matrix within the unit disk. These zeros are then used to obtain a set of linearly independent equations the solution of which gives the desired unknown coefficients. The last step consists of transform inversion once the expression for the generating function of the unknown state probabilities is explicitly written. This approach, however, has generally received poor reviews in the literature for its numerical properties. Neuts gives a set of reasons why transform techniques are algorithmically unattractive [6, pp. 29-30]:

- It is difficult to compute the zeros accurately.

- Even when accurate computation is possible the zeros are

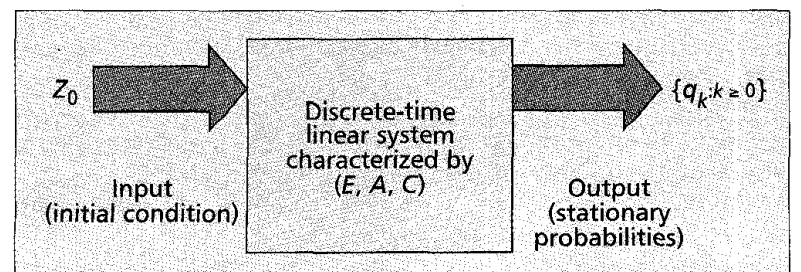

Figure 2. A queuing system reduced to a linear system. typically clustered together, and this makes the set of linear equations to be solved a very ill-conditioned one.

In addition to this problem of numerical instability, algorithms based on the transform approach are difficult to implement, and relatively slower than the alternative methods except for certain special cases.

The alternative matrix-analytic approach pioneered by Neuts [5, 6] for the $M / G / 1$ and $G / M / 1$ type Markov chains is well recognized due to its algorithmic nature and better numerical stability compared to the transform approach. The matrix-analytic methods basically consist of iterative algorithms to find the minimal nonnegative solution of certain nonlinear matrix equations arising in such chains. The stationary probabilities of interest can then be found using recursive computations. An additional advantage of these methods is the absence of transform domain matrix manipulations and transform inversion requirement. However, the low linear convergence rates of the iterative algorithms employed makes the use of this method impractical for problems of large dimensionality, especially under heavy traffic load, a condition which also increases storage requirements during recursive computation of the stationary probabilities. Moreover, to the best of our knowledge extensions of this approach to the cases of finite buffer and Markov chains with repeating rows do not exist.

Another approach is to exploit the special structure of a particular Markov chain. Although such methods provide very accurate results with reasonable execution times, they work only for the particular chain structure for which they are devised, and in most cases generalization to other queuing systems is very difficult if not impossible.

We next outline the invariant subspace approach to analyzing general queues described by the evolution equation, Eq. 1. We refer the reader to [7-9] for an extensive treatment of the approach, the algorithms therein, and relative performance of the approach in terms of execution times, stability, and robustness.

\section{THE INVARIANT SUBSPACE APPROACH}

The invariant subspace approach is algebraic, and is fairly different from the three approaches briefly discussed above. In this approach we view the problem of obtaining the stationary queue occupancy probabilities as one of determining the output of a linear, discrete-time, phase-invariant, finite-dimensional dynamic system [10], the initial conditions of which are to satisfy certain constraints. In particular, the stationary probabilities will take over the role of the output of the dynamic system, and the unknown empty queue probability will act as the unknown initial condition (or input) of the system. The determination of this unknown initial condition is discussed in the sequel.

Using the queue evolution equation, we obtain a generalized state-space representation for the described dynamic system [9],

$$
z_{k+1} E=z_{k} A, \quad q_{k}=z_{k} C,
$$

where $\left\{q_{k}: k \geq 0\right\}$ is the output, $E, A$, and $C$ are matrices of suitable size, $z_{k}$ is called the descriptor of the system, and the initial condition $z_{0}$ is to satisfy certain linear constraints. This is how the linkage between queuing systems and linear system theory is built (Fig. 2). Given the queuing model of interest, it is in general very easy to obtain the matrix parameters $E, A$, and $C$ of the associated dynamic system. 


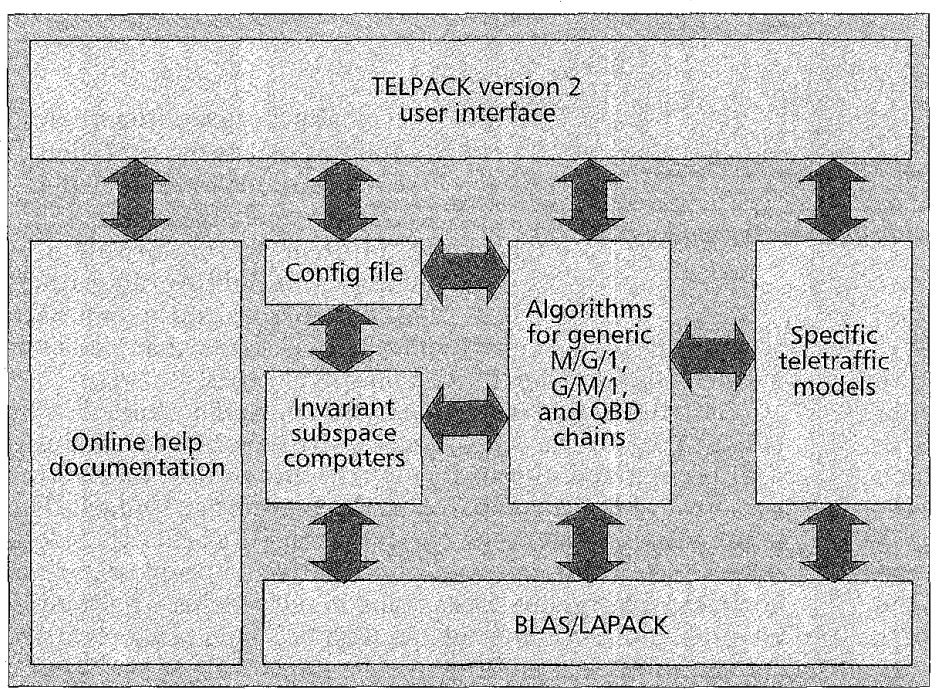

Figure 3. TELPACK software architecture.

The initial condition $z_{0}$ is determined so that no unstable mode of the generalized state-space system (or descriptor system) is excited. For this purpose, the system is decomposed into its forward and backward subsystems. Imposing the stability condition on the decomposed system, which amounts to exciting the forward subsystem only, one obtains a row vector $g$ and two matrices $F$ and $H$ of appropriate sizes so that the stationary queue occupancy probabilities can be expressed in the matrix-geometric form

$$
q_{k+N}=g F^{k} H, k \geq 0,
$$

where $N>0$ corresponds to the number of servers in the queuing model.

It is also possible to extend the above formulation to the case of finite queues. We show that the stationary solution in this case has the mixed matrix-geometric form

$$
q_{k+1}=g_{1} F_{1}^{k} H_{1}+g_{2} F_{2}^{K-k} H_{2}, \quad 0 \leq k<K,
$$

where $K$ is the size of the waiting room in the queue. Here the matrix parameters $F_{i}$ and $H_{i}, i=1,2$, are obtained by the same state-space decomposition procedure used in the case of infinite levels. The first term (second term) in the above equation can be viewed as the output of the forward subsystem (backward subsystem) obtained through the decomposition of the descriptor system. As opposed to the case of infinite queues, both subsystems can now be excited since the finite size $K$ of the waiting room eliminates the possibility of instability (i.e., queue occupancy growing without bound). The effect of $K$ comes into the picture only in obtaining the vector parameters $g_{1}$ and $g_{2}$, and hence is minimal.

The only computation-intensive part for the unified technique briefly outlined above is the forward-backward decomposition of the generalized state equations, Eq. 2. This decomposition indeed amounts to computing bases for the stable (forward) and unstable (backward) deflating or generalized invariant subspaces of the matrix pencil $\lambda E-A$, hence the name of the approach. Such invariant subspace computations can be carried out by the well-known techniques of numerical linear algebra

- (Generalized) matrix-sign function iterations

- Ordered (generalized) Schur decomposition

- Spectral divide-and-conquer methods

Both of the first two methods have been used successfully in the past to solve matrix Riccati and Lyapunov equations of optimal control and filtering [10]. The matrix-sign function can be computed by iterative algorithms with quadratic or higher convergence rates. The fact that it is also amenable to parallel implementation and the simplicity of its iterations lead to a powerful algorithm for finding the stationary solution of general queves.

Several numerical experiments we have conducted demonstrated that Schur-decompositionbased implementations of the invariant subspace approach outperform those based on serially implemented matrix-sign function iterations in terms of both execution times and numerical stability. We must note, however, that implementation of Schur decomposition is not straightforward, and one should rely on linear algebra packages that offer routines for this decomposition.

Spectral divide-and-conquer methods, on the other hand, produce very accurate results even for very ill-conditioned problems, and are also easy to implement. When compared to the previous two techniques, however, these methods are significantly slower, and should therèfore be preferred only when accuracy is of utmost importance.

As can be seen from the above discussion, the invariant subspace approach does not assume any particular Markov chain type, say $M / G / 1$ or $G / M / 1$, to proceed to the solution. It is solely built on top of the generic queuing system described by the evolution equation, Eq. 1. Although this should not dismiss minor modifications in the formulation for different chain types, and substantial simplifications are thus possible for the particular case of QBD chains, the approach in general constitutes a unifying framework to analyze finite/infinite, single/multiple-server, and continuous-/discrete-time queues. The overall method to solve for the stationary queue occupancy probabilities appears to offer significant reductions in both execution time and storage requirements compared to conventional methods. The possibility of using alternative numerical techniques to essentially do most of the job allows appreciable flexibility for making different compromises, all within the same framework, among speed, memory, and accuracy according to one's particular needs or resources. Furthermore, the simple matrix-geometric solution forms make it very easy to obtain various performance measures of interest such as overflow probabilities (or cell loss rates) and moments of queue occupancy distribution (or cell delay and cell delay variation).

\section{TELPACK}

As discussed above, the invariant subspace approach unifies all queuing systems described by the generic evolution equation, Eq. 1 , under one umbrella in the sense that their computational analyses amount essentially (time- and memory-wise) to a wellknown task, invariant subspace computation, of numerical linear algebra. This suggests that a software package could be developed around a common computational engine to analyze important queuing systems of interest to the teletraffic analysts. Our endeavor to develop TELPACK has started by this token. Currently, the first release of the package, version 1.0a [2], is publicly available via the Web at http://www.cstp.umkc.edu/org/tn/telpack, and we are working toward releasing a more advanced version with enhanced user assistance.

Putting invariant subspace computation aside, the algorithms implementing our approach for a given queuing system are mostly composed of matrix-algebraic operations.

Therefore, in developing TELPACK we relied on the library of basic linear algebra subprograms (BLAS) that offers proven successful implementations of such operations between vector and matrix objects. For higher-level matrix operations 
( $L U$ decomposition, backward substitution, inversion, QR factorization, Schur decomposition, etc.) needed during invariant subspace computations, we used the linear algebra package (LAPACK) routines which have been written so that as much of the computation as possible is performed by BLAS calls. This configuration has an advantage: highly efficient machineoptimized implementations of the BLAS exist for many modern high-performance computers, and users of TELPACK may link with their local optimized BLAS installations, if available, to enjoy substantially enhanced runtime performance. Nevertheless, TELPACK has been designed to be a self-contained package, and includes the necessary subset of BLAS and LAPACK routines as well as a detailed users' manual. All that is required to get it running is a UNIX box and a $\mathrm{C}$ compiler. The first release of the package has been tested successfully on the following UNIX platforms:

- DEC Alpha station (OSF/1 V3.2a)

- SUN Sparc station (SunOS 4.1.3 and Solaris 2.4)

- IBM Risc machine (Ultrix 4.4)

- HP 712/60 workstation (HP-UX A.09.05)

- Intel 486DX PC (Linux Kernel 2.0.0)

For information on the BLAS and LAPACK libraries, we refer the reader to [11].

TELPACK v. 1.0a focuses on generic Markov chains of types $\mathrm{M} / \mathrm{G} / 1, \mathrm{G} / \mathrm{M} / 1$, and $\mathrm{QBD}$, which are of central importance in teletraffic analysis. The support for QBD chains is the richest of all: all four combinations of finite/infinite and discrete-/continuous-time QBD chains are handled while only discrete-time infinite $M / G / 1$ and $G / M / 1$ type Markov chains are being supported. Since one single method would hardly be the best for all situations, TELPACK also offers certain refined methods based on the matrix-analytic approach for all three chain types. A quadratically convergent variant of this approach, the logarithmic reduction method [12], for infinite QBD chains is also implemented. As for implementation of the invariant subspace approach, v. 1.0a puts the emphasis on the matrix-sign function iterations, and uses Schur decomposition only for the case of infinite QBD chains.

TELPACK v. $1.0 \mathrm{a}$ has a simple text-based user interface through which users can access online help as well as browse through menus listing the supported Markov chain types and the solution methods offered for each type. The program expects users to prepare numerical input files describing the Markov chain to be solved, and writes the various computed outputs in a number of output files. Among these outputs are the stationary queue probabilities, the factorial moments of the queue occupancy distribution, an estimated error of the solution, and the key matrix and vector elements characterizing the solution (e.g., $g, F$, and $H$ ). The program also analyzes the asymptotic queue length behavior based on the computed stationary probabilities, and determines the coefficient and rate of the geometric decay. Certain critical parameters (e.g., epsilons to be used in stopping criteria in iterative algorithms) reside in two configuration files; and users, by modifying these parameters, can alter various runtime decisions as they find appropriate for their problem.

As for the next version of TELPACK, our major focus is technically on two issues:

- Using Schur decomposition also for $M / G / 1$ and $G / M / 1$ type Markov chains

- Supporting finite M/G/1 type Markov chains

More of our effort has, however, been toward providing better user assistance. That is, the next version will incorporate several widely encountered teletraffic models less generic in nature than $\mathrm{M} / \mathrm{G} / 1$ or $\mathrm{G} / \mathrm{M} / 1$ type Markov chains. Among these models are (discrete) (batch) Markovian arrival processes (D-BMAP) and phase-type (PH) processes. While the wellknown Erlangian server is an example of the latter, the Markov-modulated Poisson and Bernoulli processes (MMPP and MMBP), for example, are special cases of MAP and DMAP, respectively. Such particular models as MMPP or MMBP will enjoy individual recognition in the next version, which will rid prospective users of TELPACK of the burden of mapping their problems into the terms and format of generic chain types $\mathrm{M} / \mathrm{G} / 1, \mathrm{G} / \mathrm{M} / 1$, or QBD. Figure 3 depicts the software architecture of the next version of TELPACK.

\section{REFERENCES}

[1] W. Willinger, "Traffic modeling for high-speed networks: theory versus practice," F. P. Kelly and R. J. Williams, eds., Stochastic Networks, Berlin: Springer-Verlag, 1995.

[2] A. Agrawal et al., TELPACK V1.0a Manual, Comp. Sci. Telecommun. Univ. Missouri-Kansas City, 1997, available via http://www.cstp.umkc edu/org/tn/telpack.

[3] R. Guerin, H. Ahmadi, and M. Naghshineh, "Equivalent capacity and its application to bandwidth allocation in high-speed networks," IEEE JSAC, vol. 9, 1991, pp. 968-81.

[4] G. L. Choudhury, D. M. Lucantoni, and W. Whitt, "Squeezing the most out of ATM," IEEE Trans. Commun., vol. 44, 1996, pp. 203-17.

[5] M. F. Neuts, Structured Stochastic Matrices of $M / G / 1$ Type and Their Applications, New York: Marcel Dekker, 1989.

[6] M. F. Neuts, Matrix-Geometric Solutions in Stochastic Models, Baltimore: Johns Hopkins Univ. Press, 1981.

[7] N. Akar and K. Sohraby, "An invariant subspace approach in M/G/1 and G/M/1 type Markov chains," Commun. Stats.-Stochastic Models, vol. 13, no. 3, 1997.

[8] N. Akar, N. C. Oguz, and K. Sohraby, "A novel computational method for solving finite QBD processes," Submitted to Commun. Stats. Stochastic Models, 1997

[9] N. Akar, N. C. Oguz, and K. Sohraby, "Matrix-geometric solutions of $M / G / 1$ type Markov chains: a unifying generalized state-space approach," IEEE JSAC, vol. 16, 1998, pp. 626-39.

[10] T. Kailath, Linear Systems, Englewood Cliffs, NJ: Prentice Hall, 1980.

[11] E. Anderson et al., LAPACK User's Guide, 2nd ed., 1994; also available at http://www.netlib.org.

[12] G. Latouche and V. Ramaswami, "A logarithmic reduction algorithm for quasi-birth-death processes," J. Appl. Prob., vol. 30, pp. 650-74, 1993.

\section{BIOGRAPHIES}

NAIL AKAR (akar@sprintcorp.com) received a B.S. degree from Middle East Technical University, Ankara, Turkey, in 1987, and M.S. and Ph.D. degrees from Bilkent University, Ankara, Turkey, in 1989 and 1994, respectively, all in electrical and electronics engineering. From 1994 to 1995 he conducted research as a visiting scholar in the Computer Science Telecommunications Department at the University of Missouri-Kansas City, and in 1996 was a visiting assistant professor in the same program. Since 1997 he has been with Technology Planning and Integration, Long Distance Division, Sprint. His current research areas include queuing analysis, IP/ATM networks, flow control, routing, and pricing.

NiHAT CEM Oguz (ncoguz@cstp.umkc.edu) received a B.S. degree in electrical and electronics engineering from Middle East Technical University, Ankara, Turkey, in 1987. Thereafter, he joined Bilkent University and received his M.S. and Ph.D. degrees in 1990 and 1995, respectively, both in electrical and electronics engineering. In 1996, he joined Computer Science Telecommunications at the University of Missouri-Kansas City as a visiting scholar. He has been with the same program as a visiting assistant professor since 1997. His current research interests include network performance evaluation, loss process characterization and forward error correction in ATM networks, and parallel numerical linear algebra applications.

KHOSROW SOHRABY (sohraby@cstp.umkc.edu) is a professor with Computer Science Telecommunications, University of Missouri-Kansas City. He received B.Eng and M.Eng degrees from McGill University, Montreal, Canada, in 1979 and 1981, respectively, and a Ph.D degree in 1985 from the University of Toronto, Canada, all in electrical engineering. His current research interests include design, analysis, and control of high-speed computer and communications networks, traffic management and analysis, multimedia networks, networking aspects of wireless and mobile communications, analysis of algorithms, parallel processing, and large-scale computations. 